BULLETIN (New Series) OF THE

AMERICAN MATHEMATICAL SOCIETY

Volume 38, Number 3, Pages 293-314

S 0273-0979(01)00902-8

Article electronically published on March 27, 2001

\title{
THE ARITHMETIC AND GEOMETRY OF SALEM NUMBERS
}

\author{
EKNATH GHATE AND ERIKO HIRONAKA
}

\begin{abstract}
A Salem number is a real algebraic integer, greater than 1, with the property that all of its conjugates lie on or within the unit circle, and at least one conjugate lies on the unit circle. In this paper we survey some of the recent appearances of Salem numbers in parts of geometry and arithmetic, and discuss the possible implications for the 'minimization problem'. This is an old question in number theory which asks whether the set of Salem numbers is bounded away from 1 .
\end{abstract}

\section{Contents}

1. Introduction 293

2. Overview of the paper 295

3. Geodesics on arithmetic hyperbolic surfaces 296

3.1. Salem extensions $\quad 296$

3.2. Arithmetic Fuchsian groups $\quad 298$

3.3. Salem numbers and hyperbolic matrices $\quad 300$

3.4. Closed geodesics on hyperbolic surfaces 303

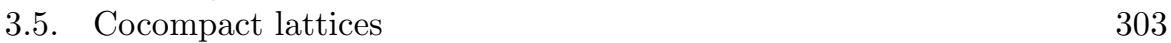

4. Growth rates and pretzel knots

\begin{tabular}{ll|l} 
4.1. Growth series of Coxeter groups & 304 \\
\hline
\end{tabular}

4.2. Arithmeticity of hyperbolic triangle groups $\quad 307$

4.3. Alexander polynomials of pretzel links $\quad 307$

5. Special values of $L$-functions $\quad 309$

5.1. Stark units and Salem numbers $\quad 309$

\begin{tabular}{lll} 
5.2. Lower bounds for relative regulators & 310 \\
\hline
\end{tabular}

5.3. Mahler measures and $L$-values $\quad 310$

6. Best results and records 311

\begin{tabular}{ll} 
References & 312 \\
\hline
\end{tabular}

\section{INTRODUCTION}

A Salem number is a real algebraic integer, greater than 1, with the property that all its conjugates lie on or within the unit circle, and at least one conjugate lies on the unit circle. This paper deals with the following unsolved problem:

Received by the editors November 20, 1999

2000 Mathematics Subject Classification. Primary 11R06, 11R52, $20 \mathrm{~F} 55$.

(C)2001 American Mathematical Society 
Problem 1 (Minimization problem). Is the set of Salem numbers bounded away from 1 ?

The minimization problem is closely related to the following question posed by Lehmer [25] in 1933.

Problem 2 (Lehmer's question). Is there a $\delta>0$ such that the Mahler measure of every irreducible monic polynomial $P(x)$ with integer coefficients is either 1 or larger than $1+\delta$ ?

The Mahler measure $M(P)$ of a monic polynomial $P(x) \in \mathbb{Z}[x]$ is the product of the absolute values of the roots of $P(x)$ which lie outside the unit circle, and is 1 if there are no such roots:

$$
M(P)=\prod_{P(\theta)=0} \max \{1,|\theta|\} .
$$

Thus $M(P) \geq 1$ and it may be checked that if $P(x)$ is monic and irreducible, then $M(P)=1$ if and only if $P(x)$ is a cyclotomic polynomial or the monomial $x$.

Of special interest to Lehmer were palindromic polynomials (also sometimes called reciprocal or symmetric polynomials): these are polynomials $P(x) \in \mathbb{Z}[x]$ that satisfy

$$
P(x)=x^{m} P(1 / x),
$$

where $m$ is the degree of $P(x)$. Equivalently, palindromic polynomials are integer polynomials that read the same whether read backwards or forwards. A nonlinear irreducible palindromic polynomial must have even degree since palindromic polynomials of odd degree always have -1 as a root. In [25], Lehmer found the monic palindromic polynomials of degrees 2, 4, 6, and 8 with smallest Mahler measure. For degree 10 and higher, the best polynomial Lehmer could find was

$$
L(x)=x^{10}+x^{9}-x^{7}-x^{6}-x^{5}-x^{4}-x^{3}+x+1,
$$

which we will refer to throughout this paper as the Lehmer polynomial. This polynomial still stands today as the polynomial with smallest known Mahler measure:

$$
M(L)=1.17628 \ldots
$$

If $P(x) \in \mathbb{Z}[x]$ is a monic, irreducible polynomial with $1<M(P)<M(L)$, then $P(X)$ must be palindromic, since Smyth has shown [40] that $1.32471 \ldots$, the unique real root of $S(x)=x^{3}-x-1$, is a lower bound for the set of Mahler measures of non-palindromic polynomials with Mahler measure strictly larger than 1.

We will refer to the minimal polynomial of a Salem number $\epsilon$ as a Salem polynomial. Its Mahler measure is clearly just $\epsilon$. It is not difficult to prove that Salem polynomials are always palindromic.

The Lehmer polynomial $L(x)$ is a Salem polynomial: it is the minimal polynomial of the Salem number $\epsilon_{L}=1.17628 \cdots=M(L)$. Thus $\epsilon_{L}$ is both the smallest known Salem number and the smallest known Mahler measure.

Flammang, Grandcolas, and Rhin [18] have shown that there are no Salem numbers less than 1.3, of degree less than 40, other than the forty-seven Salem numbers tabulated by Boyd and Mossinghoff in [5], 66 and [29]. As a consequence, if there is a Salem number smaller than $\epsilon_{L}$, it would have to have degree larger than 40 .

The purpose of this note is to expose some of the recent appearances that Salem numbers, and more generally Mahler measures, have made in arithmetic and geometry. A more detailed overview of the topics we treat is given in the next section. 
We have tried to adopt an interdisciplinary viewpoint which we hope will shed a fresh perspective on the minimization problem.

In closing this Introduction we mention that Salem numbers originally arose in the study of Fourier analysis and uniform distribution [34]. The reader can find a convenient survey of some properties of Salem numbers and those of the closely related Pisot-Vijayaraghavan numbers in [8] and [2]. Here too the reader will find a more detailed discussion of certain aspects of the minimization problem. Another area related to Mahler measures, which we do not touch upon, is the field of dynamical systems. The jumping off point here is that the entropy of certain dynamical systems attached to polynomials equals the logarithm of the Mahler measure of these polynomials. We simply refer the curious reader to the works [26, [35, [17, and [39 for further details.

Acknowledgments. We would like to thank D. Boyd, D. Lind, C. McMullen, M. Mossinghoff, P. Sarnak, J.-P. Serre, B. Sury, D. Ulmer, and T. N. Venkataramana for useful conversations and correspondence, and the members and staff of the I.H.E.S. where the research for this paper began. We are also indebted to the referee for helpful comments.

\section{OVERVIEW OF THE PAPER}

Here is a brief overview of the contents of this paper. Sections 3.1 to 3.4 describe a relation between the minimization problem for Salem numbers and a minimization problem for the lengths of closed geodesics on arithmetic hyperbolic surfaces, i.e., hyperbolic surfaces attached to arithmetic Fuchsian groups. This material was worked out by the authors, but it is certainly not new (see, for example, Neumann and Reid [30]). An equivalent formulation of these ideas in terms of lattices in semi-simple real Lie groups was first worked out by Sury in [45]. We recall this briefly in Section 3.5

Floyd and Plotnick [19] showed that some Salem numbers, including $\epsilon_{L}$ above, are equal to the asymptotic growth rates of certain hyperbolic planar reflection groups. The smallest Salem number that arises in this way is again $\epsilon_{L}$, as shown by the second author in [22. These matters are described in Section 4.1

The role of Alexander polynomials in the study of Salem numbers and the minimization problem is discussed in Section 4.3 . The second author has shown $[22$ that the Salem polynomials arising, as in Section 4.1, from polygonal reflection groups are the Alexander polynomials of certain pretzel knots. A corollary is that Lehmer's polynomial solves the minimization problem for this family of polynomials.

In Section [5 we explain how Salem numbers and Mahler measures show up in the special values of $L$-functions. We start, in Section 5.1 with an observation of Chinburg [12], who relates Salem numbers to Stark units, and hence to the values of certain (abelian) Artin $L$-functions at $s=0$. Then, in Section 5.2, we describe work and conjectures of Costa, Friedman and Skoruppa [21] on the ratios of regulators of extensions of number fields, and their relation to the minimization problem.

Pursuing this theme further, we discuss, in Section [5.3, some recent striking results of Smyth, Ray, Boyd, Deninger, Bornhorn and Rodriguez Villegas that show that the special values of more general $L$-functions are connected to the logarithms of Mahler measures of polynomials in many variables. The possible implications for the minimization problem are discussed following Boyd in [9]. 
We conclude in Section [6 with a review of some of the sharpest results known to us in the direction of solving the minimization problem. These results have been obtained by methods completely different from those described in this paper. We also refer the reader to the web page on Lehmer's Conjecture maintained by Mossinghoff [28] where, among other things, much up to date numerical information related to Lehmer's question may be found.

\section{GeOdesics on ARIThMetiC HyPERBoliC SURFACES}

In this section we show that the minimization problem for Salem numbers is equivalent to the minimization problem for the lengths of closed geodesics on arithmetic hyperbolic surfaces.

3.1. Salem extensions. We start by describing some properties of the number field $L=\mathbb{Q}(\epsilon)$ generated by a Salem number $\epsilon$.

A number field $L$ is a field extension of $\mathbb{Q}$ of finite degree over $\mathbb{Q}$. We may write $L=\mathbb{Q}(\theta)$ for an element $\theta \in L$ which generates $L$ over $\mathbb{Q}$. Consequently we may identify $L$ with $\mathbb{Q}[x] /\langle P(x)\rangle$, where $P(x) \in \mathbb{Z}[x]$ is the minimal polynomial of $\theta$. Then the degree of $L$ is the dimension of $L$ as a vector space over $\mathbb{Q}$, and equals the degree of the minimal polynomial $P(x)$.

If $\omega$ is any root of $P(x)$ ( $\omega$ is called a conjugate of $\theta$ ), then there is a unique isomorphism from $L$ to $\mathbb{Q}(\omega)$ sending $x$ to $\omega$. Thus, each root $\omega$ of $P(x)$ defines a $\mathbb{Q}$-algebra monomorphism

$$
\sigma_{\omega}: L \stackrel{\simeq}{\longrightarrow} \mathbb{Q}(\omega) \subset \mathbb{C} .
$$

Composition by complex conjugation yields another (possibly identical) embedding

$$
\overline{\sigma_{\omega}} \quad\left(=\sigma_{\bar{\omega}}\right) .
$$

A pair of such embeddings $\{\sigma, \bar{\sigma}\}$ is called an infinite place of $L$. An infinite place is real if $\sigma=\bar{\sigma}$; otherwise it is complex. In the former case we set $L_{\sigma}=\mathbb{R}$, and in the latter case we set $L_{\sigma}=\mathbb{C}$ and call $L_{\sigma}$ the completion of $L$ at the place $\sigma$. If all the infinite places of $L$ are real, then we say $L$ is a totally real number field.

Since $\mathbb{C}$ and $\mathbb{R}$ have natural topologies, each of the infinite places of $L$ induce (distinct) topologies on $L$. However there are other topologies on $L$, the nonarchimedean topologies. The natural embeddings of $L$ into the $\mathfrak{q}$-adic completions of $L$, as $\mathfrak{q}$ varies through the prime ideals of $L$,

$$
\sigma_{\mathfrak{q}}: L \rightarrow L_{\mathfrak{q}}
$$

induce such topologies on $L$. For this reason, we will refer to the primes $\mathfrak{q}$, and sometimes even to the corresponding embeddings $\sigma_{\mathfrak{q}}$, as the finite places of $L$.

Now let $\epsilon$ be a Salem number, i.e., an algebraic integer all of whose conjugates lie on or inside the unit circle with at least one on the unit circle, and let $K=\mathbb{Q}(\epsilon)$ be the number field generated by $\epsilon$. We identify $K$ with $\mathbb{Q}[x] /\langle P(x)\rangle$ where $P(x)$ is the minimal polynomial of $\epsilon$. As mentioned already in the Introduction, the degree of $K$ must be even, since $P(x)$ is irreducible and palindromic. Say this degree is $2 n$.

Note that all the conjugates of $\epsilon$, except for $\frac{1}{\epsilon}$, which is also real, lie on the unit circle. This is because if any complex root $z$ of $P(x)$ were to lie strictly within the unit circle, then the root $\frac{1}{z}$ would lie outside it, contradicting the definition of $\epsilon$. 


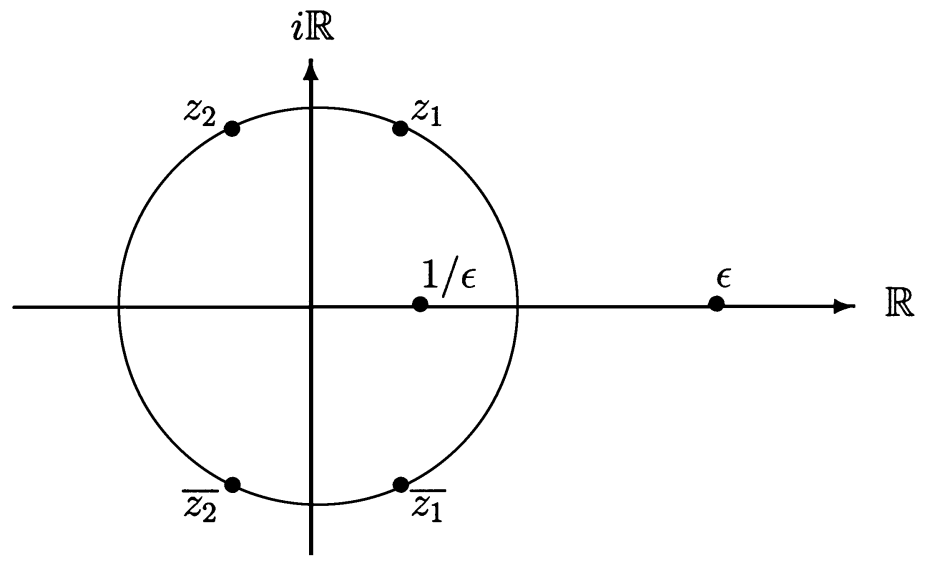

Figure 3.1. A (degree six) Salem number $\epsilon$ and its conjugates

Thus a list of the conjugates of $\epsilon$ can be written as

$$
\epsilon, \frac{1}{\epsilon}, z_{1}, \overline{z_{1}}, \ldots, z_{n-1}, \overline{z_{n-1}}
$$

where $\left|z_{i}\right|=1$, for $i=1, \ldots, n-1$. A plot of the set of conjugates of a typical Salem number $\epsilon$ is given in Figure 3.1 By the general discussion above we see that $K$ has $n-1$ complex places, say $\tau_{1}, \ldots, \tau_{n-1}$, corresponding to the roots $z_{1}, \ldots, z_{n-1}$, and two real places, say $\tau$ and $\tau^{\prime}$, corresponding to $\epsilon$ and $1 / \epsilon$ respectively.

Since $P(x)$ is monic, irreducible, and palindromic, of degree at least 4 (recall that a Salem number has at least one conjugate on the unit circle) we may further write

$$
P(x)=x^{n} Q\left(x+\frac{1}{x}\right)
$$

for some monic, irreducible polynomial $Q(x) \in \mathbb{Z}[x]$ of degree $n \geq 2$. We see that $Q(x)$ has $n$ real roots, namely $\alpha=\epsilon+\frac{1}{\epsilon}$, and $\alpha_{i}=z_{i}+\overline{z_{i}}$, for $i=1, \ldots, n-1$. Set $k=\mathbb{Q}(\alpha)$, where $\alpha=\epsilon+\frac{1}{\epsilon}$ is the unique (real) root of $Q(x)$ larger than 2 . Then $k$ is a number field of degree $n$. We will call the quadratic extension of number fields $K / k$ a Salem extension.

Note that $k$ is a totally real field. Let $\sigma$ denote the real place of $k$ corresponding to $\alpha$, and let $\sigma_{1}, \ldots, \sigma_{n-1}$ denote the other real places of $k$ corresponding to $\alpha_{1}, \ldots, \alpha_{n-1}$.

The places of $K$ and $k$ are related. Namely $\tau$ and $\tau^{\prime}$ lie over the place $\sigma$ of $k$. This means that $\sigma(k) \subset \tau(K)$ and $\sigma(k) \subset \tau^{\prime}(K)$. Similarly the places $\tau_{1}, \ldots, \tau_{n-1}$ of $K$ lie over the places $\sigma_{1}, \ldots, \sigma_{n-1}$ of $k$.

Note that $\epsilon$ is a root of the polynomial

$$
f(x)=x^{2}-\alpha x+1 .
$$

Conversely, for any real algebraic integer $a>2$ (of degree larger than 2) whose conjugates are all real and lie in $(-2,2)$, the root $e$ larger than 1 of the polynomial

$$
f(x)=x^{2}-a x+1
$$

is a Salem number. 
Now let $L$ be an arbitrary totally real field of degree $d \geq 2$ over $\mathbb{Q}$. We say that a Salem number $\epsilon$ is a Salem number over $L$ if the totally real field $k=\mathbb{Q}(\epsilon+1 / \epsilon)$ equals $L$. Let $\mathcal{S}(L)$ denote the set of all Salem numbers over $L$.

Let $\iota$ be a real place of $L$. If, in addition, the embedding $\sigma$ of $k$ coincides with the embedding $\iota$ of $L$, then we say $\epsilon$ is a Salem number over $L$ of type $\iota$. Let $\mathcal{S}(L, \iota)$ denote the set of all Salem numbers over $L$ of type $\iota$. Clearly we have $\mathcal{S}(L)=\bigcup_{\iota} \mathcal{S}(L, \iota)$, as $\iota$ varies through all the infinite places of $L$. Now let, for $d \geq 2$,

$$
\mathcal{S}_{d}=\bigcup_{L} \mathcal{S}(L),
$$

where $L$ runs over all totally real fields of degree $d$. Thus $\mathcal{S}_{d}$ is the set of all Salem numbers of degree $2 d$. We have the following easy lemma.

Lemma 3. The set $\mathcal{S}_{d}$ has a minimal element.

Proof. We show more generally that the set of Mahler measures of monic polynomials of degree $2 d$ is a discrete set, and therefore has a minimal element. Let $P(x)=\sum_{r=0}^{2 d} a_{2 d-r} x^{r} \in \mathbb{Z}[x]$ denote such a polynomial. Since $a_{r}$ is, up to sign, a sum of the products of the roots of $P(x)$ chosen $r$ at a time, we see that

$$
\left|a_{r}\right| \leq\left(\begin{array}{c}
2 d \\
r
\end{array}\right) \cdot M(P) \text {. }
$$

Consequently, there are only finitely many monic polynomials of degree $2 d$ with bounded Mahler measure. The lemma follows.

3.2. Arithmetic Fuchsian groups. A group $\Gamma$ is a Fuchsian group of the first kind if $\Gamma$ is a discrete subgroup of $\mathrm{SL}_{2}(\mathbb{R})$ and the quotient $\Gamma \backslash H$ of the upper half plane $H$ by $\Gamma$ has finite volume.

An arithmetic Fuchsian group is a Fuchsian group of the first kind which can be constructed out of a totally real number field in a manner that we shall describe in this section. More detailed treatments can be found in 37] and [49.

Let $L$ be any number field. A central simple quaternion algebra over $L$ is an associative $L$-algebra $D$ with unit such that

- the center of $D$ is $L$,

- the only two sided ideals of $D$ are the trivial ideal and $D$ itself, and,

- the dimension of $D$ over $L$ is 4 .

It is a consequence of a general theorem of Wedderburn 23 that any such algebra is either a division algebra (that is, $D$ has no zero divisors) or $D$ is isomorphic to $\mathrm{M}_{2}(L)$, the algebra of 2 by 2 matrices over $L$.

$D$ can be equipped with the reduced trace and reduced norm maps:

$$
\text { red } \operatorname{Tr}: D \rightarrow L, \quad \text { and, } \quad \operatorname{red~} \mathrm{Nm}: D^{\times} \rightarrow L^{\times},
$$

defined as follows. Fix an extension $F$ of $L$ that splits $D$. This means there is an isomorphism

$$
h: D \otimes_{L} F \stackrel{\sim}{\rightarrow} \mathrm{M}_{2}(F) .
$$

Such an $F$ always exists. Now define

$$
\operatorname{red} \operatorname{Tr}(x)=\operatorname{trace} h(x) \text { and } \operatorname{red} \operatorname{Nm}(x)=\operatorname{det} h(x) .
$$


One checks that, so defined, red $\operatorname{Tr}$ and red $\mathrm{Nm}$ take values in $L \subset F$, and that their definition is independent of the choice of $F$ and $h$.

We say that $D$ is unramified or split at a place

$$
\sigma: L \hookrightarrow L_{\sigma}
$$

of $L$, which may be either finite or infinite, if the tensor product,

$$
D \otimes_{L, \sigma} L_{\sigma},
$$

viewed as a central simple quaternion algebra over $L_{\sigma}$, is isomorphic to $\mathrm{M}_{2}\left(L_{\sigma}\right)$. Otherwise $D \otimes_{L, \sigma} L_{\sigma}$ is a division algebra, and, in this case, we say that $D$ is ramified at $\sigma$.

If $\sigma$ is a complex place, then $D$ is necessarily split at $\sigma$. If $\sigma$ is a real place, then $D$ may ramify at $\sigma$, in which case

$$
D \otimes_{L, \sigma} \mathbb{R} \simeq \mathbb{H}
$$

is the usual Hamilton algebra of quaternions over $\mathbb{R}$.

An important property of the quaternion algebras $D$ in this context is that every such $D$ is ramified at an even number of non-complex places. Conversely, for any finite collection $\Sigma$ of non-complex places of $L$, of even cardinality, there is a unique (up to isomorphism) central simple quaternion algebra $D$ over $L$ which is ramified at exactly the places in $\Sigma$. In particular, if $D$ is unramified everywhere $(\Sigma=\emptyset)$, then $D$ is isomorphic to $\mathrm{M}_{2}(L)$.

Remark. Much of the above discussion can be described very elegantly by the following exact sequence from class field theory (see for example [38], Chapter 10, or [1]):

$$
0 \rightarrow \operatorname{Br}(L) \rightarrow \bigoplus_{\sigma} \operatorname{Br}\left(L_{\sigma}\right) \rightarrow \mathbb{Q} / \mathbb{Z} \rightarrow 0,
$$

where the sum runs over all places $\sigma$, both finite and infinite, of $L$. Here $\operatorname{Br}(L)$, respectively $\operatorname{Br}\left(L_{\sigma}\right)$, denotes the Brauer group of $L$, respectively of $L_{\sigma}$. We make this remark simply to orient the more knowledgeable reader. In essence all the information contained in (2) that we shall need in the sequel has already been discussed above in words.

Now suppose that $L$ is totally real, with infinite places $\iota_{1}, \ldots, \iota_{d}$. Fix a place $\iota$ $\left(=\iota_{j}\right.$ for some $\left.j=1, \ldots, d\right)$ of $L$. We would like to impose the following condition on the 'ramification of $D$ at $\infty$ '. We assume that $D$ is unramified at a single infinite place $\iota$ and ramified at all other infinite places. That is, we assume that

$$
D \otimes_{\mathbb{Q}} \mathbb{R}=\bigoplus_{r=1}^{d} D \otimes_{L, \iota_{r}} \mathbb{R}=\mathbb{H} \oplus \cdots \oplus \mathbb{H} \oplus \mathrm{M}_{2}(\mathbb{R}) \oplus \mathbb{H} \oplus \cdots \oplus \mathbb{H},
$$

with the single summand $\mathrm{M}_{2}(\mathbb{R})$ corresponding to the place $\iota$, and $d-1$ copies of the Hamilton algebra $\mathbb{H}$ corresponding to the other places.

An order $\mathcal{O}$ in $D$ is a subring of $D$ such that

- $\mathcal{O}$ contains the ring $\mathcal{O}_{L}$ of integers of $L$, and,

- $\mathcal{O} \otimes_{\mathcal{O}_{L}} L=D$.

A maximal order is an order which is not properly contained in any other. Let $\mathcal{O}$ be a maximal order in $D$ and let $U$ be the set of unit elements of $\mathcal{O}$ of reduced norm one. 
When $L$ is totally real, and $D$ has ramification at $\infty$ given by (3), we let $\Gamma(D, \mathcal{O}, \iota) \subset \mathrm{SL}_{2}(\mathbb{R})$ be the image of $U$ under the embedding $\iota$ fixed above. It turns out that $\Gamma(D, \mathcal{O}, \iota)$ is a discrete subgroup of $\mathrm{SL}_{2}(\mathbb{R})$, and that the volume of the quotient surface $\Gamma(D, \mathcal{O}, \iota) \backslash H$ is finite. Thus $\Gamma(D, \mathcal{O}, \iota)$ is a Fuchsian group of the first kind.

A Fuchsian group of the form $\Gamma(D, \mathcal{O}, \iota)$ is called an arithmetic Fuchsian group. The corresponding quotient surface $\Gamma(D, \mathcal{O}, \iota) \backslash H$ is called an arithmetic hyperbolic surface. A Fuchsian group of the first kind is said to be cocompact if the corresponding quotient surface is compact. An arithmetic Fuchsian group $\Gamma(D, \mathcal{O}, \iota)$ is cocompact if the defining totally real number field $L$ is not equal to $\mathbb{Q}$, that is, $d \geq 2$.

The groups $\Gamma(D, \mathcal{O}, \iota)$ do not depend on the choice of $\mathcal{O}$ up to conjugation by an element of $\mathrm{SL}_{2}(\mathbb{R})$. Indeed, although there is more than one maximal order in $D$, since $D$ is split at one infinite place, all its maximal orders are conjugate to each other. Thus, the corresponding Fuchsian group is determined up to inner automorphism of $\mathrm{SL}_{2}(\mathbb{R})$.

In view of the remarks at the beginning of this section, if the degree $d$ of $L$ is odd, there is a unique $D$ (up to isomorphism over $L$ ) which is unramified at all the finite places and is ramified at all infinite places except at $\iota$. We will denote this $D$ by $D_{\phi}$. On the other hand, if $d$ is even, then a choice of a finite place $p$ of $L$ will again give a canonical choice of $D$ (up to isomorphism), which is unramified at all the finite places except $p$ and ramified at all the infinite places except $\iota$. We shall denote this $D$ by $D_{p}$ to show that it depends on the prime $p$ of $L$. The corresponding arithmetic Fuchsian groups are denoted by $\Gamma\left(D_{\phi}, \mathcal{O}, \iota\right)$ and $\Gamma\left(D_{p}, \mathcal{O}, \iota\right)$ respectively.

3.3. Salem numbers and hyperbolic matrices. In this section we show how Salem numbers are closely related to the eigenvalues of hyperbolic elements in cocompact arithmetic Fuchsian groups.

Let $\Gamma \subset \mathrm{SL}_{2}(\mathbb{R})$ be an arbitrary Fuchsian group of the first kind. An element $\gamma \in \Gamma$ is said to be hyperbolic if its eigenvalues are distinct and real. Let $f_{\gamma}(x)$ be the characteristic polynomial of $\gamma$. Then since $\operatorname{det}(\gamma)=1$, we have

$$
f_{\gamma}(x)=x^{2}-\operatorname{tr}(\gamma) x+1
$$

Thus, $\gamma$ is hyperbolic if and only if $|\operatorname{tr}(\gamma)|>2$. Moreover, in this case the roots of $f_{\gamma}(x)$ form a pair of positive real reciprocal numbers. Let $\epsilon(\gamma)$ be the root which is larger than 1 , and let

$$
N(\gamma)=\epsilon(\gamma)^{2}
$$

be the norm of $\gamma \in \Gamma$.

Fix a totally real field $L$ of degree $d \geq 2$, and an infinite place $\iota$ of $L$. Let $\Gamma=\Gamma(D, \mathcal{O}, \iota)$ be a (cocompact) arithmetic Fuchsian group, for some central simple quaternion division algebra $D$ over $L$, and some maximal order $\mathcal{O}$ of $D$, as constructed in Section 3.2 We have the following proposition:

Proposition 4. If $\gamma=\iota(u) \in \Gamma(D, \mathcal{O}, \iota)$ is not equal to the identity or its negative, then the conjugates of $\operatorname{trace}(u)$ in $L$ lie in the interval $(-2,2)$.

Proof. By construction (see Section 3.2) the conjugates $\iota^{\prime}(u)$ of $u$ (for $\iota^{\prime} \neq \iota$ ) lie in the group of norm one units of the Hamilton quaternion algebra over $\mathbb{R}$. Thus, they are of the form

$$
\iota^{\prime}(u)=a+b i+c j+d k,
$$


with $a, b, c$ and $d \in \mathbb{R}$, and with

$$
\operatorname{norm}\left(\iota^{\prime}(u)\right)=a^{2}+b^{2}+c^{2}+d^{2}=1 .
$$

Since $a$ is real, we see that $|a| \leq 1$, and hence $\left|\iota^{\prime}(\operatorname{red} \operatorname{Tr}(u))\right|=\left|\operatorname{trace}\left(\iota^{\prime}(u)\right)\right|=$ $|2 a| \leq 2$. If $|a|=1$, we have $\iota^{\prime}(u)= \pm 1$, and hence $u$, and therefore $\gamma$, is either the identity or its negative. The proposition follows.

Proposition 5. Let $\Gamma=\Gamma(D, \mathcal{O}, \iota)$ be a cocompact arithmetic Fuchsian group, and let $\gamma \in \Gamma$ be a hyperbolic element. Then $\epsilon(\gamma)$ is a Salem number.

Remark. In fact we show that $\epsilon(\gamma)$ is the image of a Salem number $\epsilon$ under the unique real infinite place of $\mathbb{Q}(\epsilon)$ which realizes $\epsilon$ as a real number larger than 1 . Thus here, and sometimes in the sequel, we identify a Salem number with its image under such an embedding.

Proof. Assume that $\gamma=\iota(u)$. By Proposition 4 above, $\alpha=\operatorname{red} \operatorname{Tr}(u)$ is an integer in $L$, with $\iota(\alpha)>2$, and with all other conjugates lying in $(-2,2)$. Thus, since $\epsilon(\gamma)$ is the larger root of

$$
f_{\gamma}(x)=x^{2}-\iota(\alpha) x+1
$$

$\epsilon(\gamma)$ is a Salem number.

Let us define $\mathcal{S}(\Gamma(D, \mathcal{O}, \iota))$ to be the set of those Salem numbers $\epsilon$ satisfying:

- $\epsilon$ is the larger eigenvalue of a hyperbolic element in $\Gamma(D, \mathcal{O}, \iota)$, and,

- the degree of $\epsilon$ is $2 d$, that is, $\alpha=\epsilon+1 / \epsilon$ generates $L$.

Note that the set $\mathcal{S}(\Gamma(D, \mathcal{O}, \iota))$ does not depend on the choice of the maximal order $\mathcal{O}$.

In Section 3.1 we had defined $\mathcal{S}(L, \iota)$ as the set of all Salem numbers over $L$ of type $\iota$. Proposition 5 shows that

$$
\mathcal{S}(\Gamma(D, \mathcal{O}, \iota)) \subset \mathcal{S}(L, \iota) .
$$

We now ask whether it is possible to capture the entire set $\mathcal{S}(L, \iota)$ by one division algebra. When the degree $d$ of $L$ is odd, this is indeed possible:

Proposition 6. Assume that $d$ is odd, and that $D_{\phi}$ is defined as in the end of Section 3.2, Then

$$
\mathcal{S}(L, \iota)=\mathcal{S}\left(\Gamma\left(D_{\phi}, \mathcal{O}, \iota\right)\right)
$$

Proof. One containment has been shown. To show the other, let $\epsilon \in \mathcal{S}(L, \iota)$. Let $K / k$ be the corresponding Salem extension (see Section 3.1 for the definition of a Salem extension, and for notation that we shall use below). We have $k=L, n=d$, and $\sigma=\iota$. We claim that $K$ is isomorphic to a maximal subfield of $D_{\phi}$. A general theorem about division algebras shows that it suffices to check that $K$ splits $D_{\phi}$. By the remarks in Section 3.2 applied to $K$, we see that it is enough to check that each completion $K_{\wp}$ of $K$ (finite or infinite) splits $D_{\phi} \otimes_{k} K$. But

$$
D_{\phi} \otimes_{k} K \otimes_{K} K_{\wp}=D_{\phi} \otimes_{k} k_{p} \otimes_{k_{p}} K_{\wp},
$$

where $p=\wp \cap \mathcal{O}_{k}$ is the prime of $k$ lying under the prime $\wp$ of $K$. By hypothesis, $D_{\phi}$ is already split by $k_{p}$ for finite $p$, so there is nothing to check at the finite places. At the infinite places, a similar phenomena occurs at $\sigma$. As for the other infinite places $\sigma_{i}(i=1, \ldots, n-1)$, we just note that the Hamilton algebra is split by the complex numbers. 
Thus, $K$ splits $D_{\phi}$, and so the Salem extension $K / k$ sits inside $D$. Since the Salem number $\epsilon$ generates $K$, it gives an element $\gamma_{\epsilon} \in \Gamma\left(D_{\phi}, \mathcal{O}, \iota\right)$, for some maximal order $\mathcal{O} \subset D$. Note that $\gamma_{\epsilon}$ is hyperbolic, since its trace is just $\sigma\left(\operatorname{tr}_{K / k}(\epsilon)\right)=\sigma\left(\epsilon+\frac{1}{\epsilon}\right)=$ $\sigma(\alpha)>2$. Clearly the larger eigenvalue $\epsilon\left(\gamma_{\epsilon}\right)$ of $\gamma_{\epsilon}$ is just $\tau(\epsilon)$. Finally the degree of $\epsilon$ is $2 d$. This shows the other inclusion.

When the degree $d$ of $L$ is even, $\mathcal{S}(L, \iota)$ may not be equal to $\mathcal{S}(\Gamma(D, \mathcal{O}, \iota))$ for one particular $D$. First we introduce some terminology. Let $K / k$ be an arbitrary quadratic extension of number fields. A prime ideal $p$ of $k$ is said to be inert in $K$ if the ideal $\wp$ generated by $p$ in $\mathcal{O}_{K}$ is a prime ideal of $K$. Also, $p$ is said to split in $K$ if the ideal generated by $p$ in $\mathcal{O}_{K}$ factors as a product of two distinct prime ideals of $K$.

Let us now assume that $\epsilon \in \mathcal{S}(L, \iota)$. Let $K / k$ denote the Salem extension corresponding to $\epsilon$. Fix a division algebra $D$ over $L$ with ramification as in (3). Let $\Sigma_{f}(D)$ denote the set of finite places of $D$ where $D$ is ramified. Recall that $\Sigma_{f}(D)$ is a finite set of odd cardinality. The following proposition gives a criterion to check whether $\epsilon$ is the (larger) eigenvalue of a hyperbolic matrix in $\Gamma(D, \mathcal{O}, \iota)$.

Proposition 7. Assume that d is even.

1. If for each $p \in \Sigma_{f}(D), p$ is inert in $K$, then $K$ sits inside $D$.

2. If $K$ sits inside $D$, then for all $p \in \Sigma_{f}(D), p$ does not split in $K$.

Proof. The proof of the first statement of Proposition 7 is exactly the same as the proof of Proposition 6, except that, additionally, for each $p \in \Sigma_{f}$, we have to check the splitting of $D_{p} \otimes_{k} K$ at $K_{\wp}$, where $\wp$ is the prime of $K$ generated by $p$. But since

$$
D_{p} \otimes_{k} K \otimes_{K} K_{\wp}=D_{p} \otimes_{k} k_{p} \otimes_{k_{p}} K_{\wp}
$$

it suffices to show that the extension $K_{\wp} / k_{p}$ splits $D_{p} \otimes_{k} k_{p}$. But this follows from the general fact that over a local field, a quaternion algebra is always split by the unique unramified extension of degree 2 (see 38, Chapter 12).

For the second statement, fix $p \in \Sigma_{f}$. If $p=\wp \wp^{\prime}$ splits in $K$ and $K$ sits in $D$, then $D \otimes_{k} k_{p}$ would contain the subalgebra $K \otimes_{k} k_{p}=K_{\wp} \times K_{\wp^{\prime}}$ which has zero divisors. This is a contradiction.

Proposition 7 shows that, when the degree $d$ of $L$ is even, we may not be able to find a single, or even finitely many, division algebras $D$ which account for all Salem numbers over $L$ (of type $\iota$ ). We must be content with the following result:

Corollary 8. Assume $d$ is even, and let $D_{p}$ be the division algebra over $L$ defined as in the end of Section 3.2. Then

$$
\mathcal{S}(L, \iota)=\bigcup_{p} \mathcal{S}\left(\Gamma\left(D_{p}, \mathcal{O}, \iota\right)\right)=\bigcup_{D} \mathcal{S}(\Gamma(D, \mathcal{O}, \iota)),
$$

where $p$ varies through all finite places of $L$, and $D$ varies over all division algebras over $L$ that are ramified at exactly one infinite place.

Proof. Let $\epsilon \in \mathcal{S}(L, \iota)$, and let $K / k$ denote the corresponding Salem extension. Choose a prime $p$ of $k$ such that $p$ is inert in $K$. Then, by Proposition [7, $\epsilon \in$ $\mathcal{S}\left(\Gamma\left(D_{p}, \mathcal{O}, \iota\right)\right)$. All the other containments have already been shown or are obvious. 
3.4. Closed geodesics on hyperbolic surfaces. Let $\Gamma$ be a Fuchsian group of the first kind. In this section we describe some properties of the quotient surface $\Gamma \backslash H$, and construct a bijection between the conjugacy classes of hyperbolic elements in $\Gamma$ and closed geodesics on $\Gamma \backslash H$. As a consequence we deduce that the minimization problem for Salem numbers is equivalent to the minimization problem for the lengths of closed geodesics on arithmetic hyperbolic surfaces.

So let $\Gamma \subset \mathrm{SL}_{2}(\mathbb{R})$ be an arbitrary Fuchsian group of the first kind. Recall that a matrix $\gamma \in \Gamma$ is hyperbolic if and only if $|\operatorname{tr}(\gamma)|>2$. We say that a matrix $\gamma \in \Gamma$ is elliptic, respectively parabolic, if $|\operatorname{tr}(\gamma)|<2$, respectively $|\operatorname{tr}(\gamma)|=2$. As a transformation of $\mathbb{C} \cup \infty, \gamma$ has the following properties (see [49], page 115):

$$
\begin{aligned}
\gamma \text { is hyperbolic } & \Longleftrightarrow \gamma \text { has two distinct fixed points in } \mathbb{R} \cup \infty, \\
\gamma \text { is elliptic } & \Longleftrightarrow\left\{\begin{array}{r}
\gamma \text { has two distinct fixed points of the form } \\
z, \bar{z}, \text { for } z \in \mathbb{C} \text {, and, }
\end{array}\right. \\
\gamma \text { is parabolic } & \Longleftrightarrow \gamma \text { has a unique fixed point in } \mathbb{R} \cup \infty .
\end{aligned}
$$

In general the induced hyperbolic structure makes the quotient space $\Gamma \backslash H$ an orbifold, with orbifold singularities corresponding to fixed points of elliptic elements. The group $\Gamma$ is cocompact (i.e. the orbifold quotient is compact) if and only if $\Gamma$ does not have any parabolic elements.

Proposition 9. Let $\Gamma$ be a Fuchsian group of the first kind. Then there is a bijection between conjugacy classes of hyperbolic elements $\gamma \in \Gamma$ and closed geodesics $g(\gamma)$ on $\Gamma \backslash H$. In this correspondence, the length of $g(\gamma)$ is $\log N(\gamma)$.

Proof. Suppose that $\gamma \in \Gamma$ is hyperbolic. Then $\gamma$ has two distinct fixed points on $\mathbb{R} \cup \infty$. Let $G$ be the geodesic in $H$ which joins them. Then, for each point $P$ on $G$, the point $\gamma(P)$ also lies on $G$, and some simple integration shows that the distance between $P$ and $\gamma(P)$ is $\log N(\gamma)$ (see [49], Proposition 2.8, for more details). Let us call $G(\gamma, P)$ the (open) geodesic joining $P$ and $\gamma(P)$ in $H$. This projects down to a closed geodesic $g(\gamma)$ on $\Gamma \backslash H$ of length $\log N(\gamma)$, which does not depend on the choice of $P$.

Conversely, let $g$ be a closed geodesic on $\Gamma \backslash H$, and let $G$ be a preimage in $H$. Then $G$ is a geodesic on $H$ and its stabilizer under the action of $\Gamma$ must contain a hyperbolic element $\gamma$ (see [48]). For this $\gamma$, we have $g=g(\gamma)$.

The following theorem follows immediately from Proposition 9 applied to the cocompact arithmetic Fuchsian groups $\Gamma=\Gamma(D, \mathcal{O}, \iota)$ constructed in Section 3.2.

Theorem 10. Let $\Gamma=\Gamma(D, \mathcal{O}, \iota)$ be a cocompact arithemetic Fuchsian group. Let $l$ be the length of a closed geodesic on the quotient surface $\Gamma \backslash H$. Then $\exp (l / 2)$ is a Salem number. Conversely, every Salem number is of this form.

An immediate consequence is that the problem of the existence of a minimal Salem number (cf. Problem 1) is actually equivalent to the following conjecture:

Conjecture 11 (Minimization problem for geodesics). There is a geodesic of minimal length amongst all closed geodesics on all arithmetic hyperbolic surfaces.

3.5. Cocompact lattices. In this section we present a simple reformulation of the previous sections in terms of lattices in semi-simple Lie groups.

Theorem 12 (Sury [44], [45]). The set of Salem numbers is bounded away from 1 if and only if there is a neighborhood of the identity element $U \subset \mathrm{SL}_{2}(\mathbb{R})$, such that 
for all cocompact arithmetic Fuchsian groups $\Gamma$, the intersection $\Gamma \cap U$ consists only of elements of finite order.

Proof. We have already seen that the problem of minimizing Salem numbers $\epsilon$ is equivalent to the problem of minimizing the traces $\epsilon+1 / \epsilon$ of hyperbolic elements in arithmetic Fuchsian groups. Since hyperbolic matrices have infinite order, the existence of the neighborhood $U$ is equivalent to the statement that the set of these traces is bounded away from 1 .

T. N. Venkataramana has pointed out to us that a positive answer to (the more general) Problem 2 implies the following conjecture:

Conjecture 13 (Margulis [27). Let $G$ be a connected semi-simple group over $\mathbb{R}$. Suppose that $\operatorname{rank}_{\mathbb{R}}(G) \geq 2$. Then there is a neighborhood $U \subset G(\mathbb{R})$ of the identity such that for any irreducible cocompact lattice $\Gamma \subset G(\mathbb{R})$, the intersection $\Gamma \cap U$ consists only of elements of finite order.

We refer the reader to [27. (especially page 322) for the definitions of terms used in this conjecture, and for its connection with Problem 2 .

\section{Growth Rates And PRetzel Knots}

In this section we discuss a restricted class of Salem numbers that arise both as growth rates for Coxeter groups and as Mahler measures of Alexander polynomials of knots. We solve the minimization problem for this restricted class of Salem numbers (see Theorem 15 and Corollary 20).

4.1. Growth series of Coxeter groups. Let $G$ be any group and $S$ a collection of generators for $G$. The growth series for $G$ with respect to $S$ is the formal power series

$$
f(x)=\sum_{n=1}^{\infty} N_{S}(n) x^{n},
$$

where $N_{S}(n)$ is the number of elements in $G$ that can be expressed minimally as a word of length $n$ in the set of generators $S$. The quantity

$$
\lim _{n \rightarrow \infty} N_{S}(n)^{1 / n},
$$

which is the reciprocal of the radius of convergence of $f(x)$, is called the asymptotic growth rate of the group $G$ with respect to the generators $S$.

We will call a group $G$ a (planar) Coxeter group if it is a discrete subgroup of the group of isometries of the spherical, hyperbolic or Euclidean plane generated by a finite set of reflections through geodesic lines (see [14). The set $S$ of generating reflections is called the set of standard generators of the Coxeter group. We will restrict our discussion to those Coxeter groups whose corresponding quotient space is compact.

Steinberg [4] showed that if $G$ is a Coxeter group and $S$ is a standard set of generators, then the corresponding growth series $f(x)$ is a rational function of $x$ (cf. [4]). Floyd and Plotnick ([19, page 503), expanding on Cannon's work [10, show that for any group $G$ of Euclidean, spherical, or hyperbolic planar isometries, the growth series with respect to suitable 'geometric' generators is a reciprocal or anti-reciprocal function:

$$
f(x)= \pm f\left(\frac{1}{x}\right),
$$


up to a factor of $(1-x)$ in some mild exceptional cases. In particular they show that for a Coxeter group $(G, S)$, if $f(x)$ is written as a quotient of relatively prime polynomials, then the denominator $\Delta(x)$ of $f(x)$ is a palindromic polynomial.

Specifically, consider the Coxeter reflection group $G_{p_{1}, \ldots, p_{d}}$ generated by reflections through the sides of a spherical, hyperbolic, or planar polygon whose interior angles are

$$
\frac{\pi}{p_{1}}, \ldots, \frac{\pi}{p_{d}}
$$

where $p_{1}, \ldots, p_{d}$ are any positive integers. Then (see [14], p. 55) $G_{p_{1}, \ldots, p_{d}}$ has the presentation

$$
G_{p_{1}, \ldots, p_{d}}=\left\langle g_{1}, \ldots, g_{d} \mid\left(g_{i}\right)^{2}=1,\left(g_{i} g_{i+1}\right)^{p_{i}}=1\right\rangle .
$$

The orbifold Euler characteristic of the quotient surface is given by

$$
\chi\left(G_{p_{1}, \ldots, p_{d}}\right)=\frac{1}{p_{1}}+\cdots+\frac{1}{p_{d}}-d+2 .
$$

The sign of $\chi(G)$, for $G=G_{p_{1}, \ldots, p_{d}}$, determines whether or not the associated planar polygon lives in the hyperbolic, Euclidean, or spherical plane. If

- $\chi(G)<0$, then $G$ is hyperbolic,

- $\chi(G)=0$, then $G$ is Euclidean, and,

- $\chi(G)>0$, then $G$ is spherical.

Of particular interest to us will be the $(p, q, r)$-hyperbolic triangle groups. These are groups of the form $G_{p, q, r}$ with

$$
\frac{1}{p}+\frac{1}{q}+\frac{1}{r}<1
$$

A picture of the fundamental domain for a $(p, q, r)$-hyperbolic triangle group is given in Figure 4.1.

Let $\Delta_{p_{1}, \ldots, p_{d}}(x)$ be the denominator of the growth series $f(x)$ of $G_{p_{1}, \ldots, p_{d}}$. For any positive integer $n$, let

$$
[n]=1+x+\cdots+x^{n-1} .
$$

Theorem 14 (Cannon-Wagreich [10, Floyd-Plotnick [19], Parry [31]). The polynomial $\Delta_{p_{1}, \ldots, p_{d}}(x)$ is given by

$$
\Delta_{p_{1}, \ldots, p_{d}}(x)=\left[p_{1}\right] \ldots\left[p_{d}\right](x-d+1)+\sum_{i=1}^{d}\left[p_{1}\right] \ldots \widehat{\left.p_{i}\right]} \ldots\left[p_{d}\right] .
$$

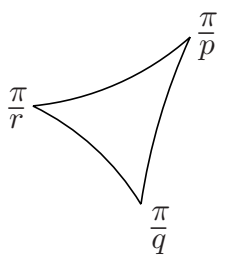

FiguRE 4.1. Fundamental domain of a $(p, q, r)$-hyperbolic triangle group 
Furthermore, $\Delta_{p_{1}, \ldots, p_{d}}(x)$ is a product of cyclotomic polynomials and at most one Salem polynomial. The Salem polynomial occurs if and only if $G_{p_{1}, \ldots, p_{d}}$ is hyperbolic, that is,

$$
\frac{1}{p_{1}}+\cdots+\frac{1}{p_{d}}<d-2 .
$$

It follows that the asymptotic growth rate of a hyperbolic Coxeter reflection group with respect to the standard generators is a Salem number.

The polynomials with smallest Mahler measure of degrees 2, 4, 6 and 8 (found by Lehmer in [25]) all arise as factors of $\Delta_{p_{1}, \ldots, p_{d}}$ for some positive integers $p_{1}, \ldots, p_{d}$ (see [22], Section 3). Also the Lehmer polynomial $L(x)=\Delta_{2,3,7}(x)$ is the denominator of the growth series of the $(2,3,7)$-hyperbolic triangle group.

As the next result shows, the minimization problem has been solved for the family of polynomials $\Delta_{p_{1}, \ldots, p_{d}}(x)$.

Theorem 15 (Hironaka [22]). Lehmer's Salem number $\epsilon_{L}$ is the smallest Salem number arising as a root of $\Delta_{p_{1}, \ldots, p_{d}}(x)$, where $p_{1}, \ldots, p_{d}$ are any positive integers.

Theorem [15 is proved by analyzing the shape of the real graph of the polynomial $P(x)=\Delta_{p_{1}, \ldots, p_{d}}(x)$ when the corresponding Coxeter reflection group $G_{p_{1}, \ldots, p_{d}}$ is hyperbolic. This graph, of which the graph of the Lehmer polynomial shown in Figure 4.2 is a typical example, has the property that $P(0)=1$ and $P(1)<$ 0 . Further, being the graph of a Salem polynomial, it crosses the $x$-axis once in the interval $[0,1]$ at the point $1 / \epsilon$ where $\epsilon$ is the corresponding Salem number. One shows (see [22] for further details) that for this particular family of Salem polynomials the distance between $1 / \epsilon$ and 1 is related to the absolute value of $\chi\left(G_{p_{1}, \ldots, p_{d}}\right)$ and so is minimized by the (2,3,7)-hyperbolic triangle group.

A suggestive coincidence is that the $(2,3,7)$-hyperbolic triangle also has the smallest volume among hyperbolic polygons. This leads to the question:

Problem 16. Is there a direct connection between the volumes of hyperbolic polygons and the asymptotic growth rates of the underlying Coxeter reflection groups?

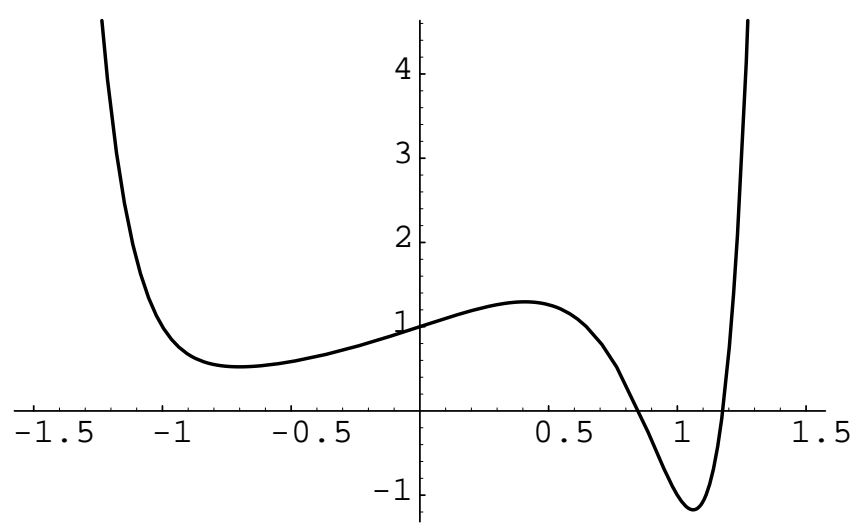

FiguRE 4.2. Real graph of the Lehmer polynomial 
4.2. Arithmeticity of hyperbolic triangle groups. We will call two discrete groups $\Gamma, \Gamma^{\prime} \subset \mathrm{SL}_{2}(\mathbb{R})$ commensurable if $\Gamma \cap \Gamma^{\prime}$ has finite index in both $\Gamma$ and $\Gamma^{\prime}$. In this section we will broaden the definition of an arithmetic Fuchsian group given in Section[3.2 to include those Fuchsian groups which, after conjugation in $\mathrm{SL}_{2}(\mathbb{R})$, are commensurable with groups of the form $\Gamma(D, \mathcal{O}, \iota)$.

Takeuchi has shown 46] that there are exactly 85 hyperbolic triangle groups $G_{p, q, r}$ which are arithmetic. (These groups are defined over only 19 totally real fields.) Included among these is the triangle group $G_{2,3,7}$.

Recall that the asymptotic growth rate of $G_{2,3,7}$ is equal to $\epsilon_{L}$, Lehmer's Salem number. Naively, one might hope that the arithmetic Fuchsian group $G_{2,3,7}$ is isomorphic to one giving rise to $\epsilon_{L}$.

This is, however, not the case. Indeed, in 46, Takeuchi shows that the $(2,3,7)$ hyperbolic triangle group is commensurable to an arithmetic Fuchsian group associated to the totally real field $k_{2,3,7}:=\mathbb{Q}\left(\cos \left(\frac{\pi}{7}\right)\right)$. The degree of $k_{2,3,7}$ over $\mathbb{Q}$ is 6 , since it is a quadratic extension of the cubic field $\mathbb{Q}\left(\cos \left(\frac{2 \pi}{7}\right)\right)$. (This latter field is cubic since it has degree equal to half the degree of the cyclotomic field generated by a primitive 7 th root of unity.) On the other hand, the totally real field $k_{L}:=\mathbb{Q}\left(\epsilon_{L}+1 / \epsilon_{L}\right)$ associated to Lehmer's Salem number $\epsilon_{L}$ has degree 5 (half the degree of the Lehmer polynomial).

Comparing degrees shows that $\epsilon_{L}$ is not quadratic over $k_{2,3,7}$, and in particular, $\epsilon_{L}$ cannot appear, as described in Section [3.3, as the larger eigenvalue of a hyperbolic matrix in $G_{2,3,7}$. Since $\epsilon_{L}$ does appear as the larger eigenvalue of a hyperbolic matrix in $\Gamma=\Gamma\left(D_{\phi}, \mathcal{O}, \iota\right)$, the arithmetic Fuchsian group attached to the division algebra $D_{\phi}$ over the totally real field $k_{L}$ (see Section 3 for an explanation of the notation), we see that $G_{2,3,7}$ and $\Gamma$ are not isomorphic subgroups of $\mathrm{SL}_{2}(\mathbb{R})$.

4.3. Alexander polynomials of pretzel links. The Alexander polynomial is a standard invariant in $\mathbb{Z}[x]$ of an oriented knot or link $L$. Let us describe it briefly: more detailed descriptions can be found in, for example, 20] or [33. Since we are interested in the Mahler measures of Alexander polynomials we only describe the Alexander polynomial up to multiplication by a non-zero rational number. Let $M=S^{3} \backslash L$ be the complement of $L$ in $S^{3}$. A choice of orientations on the components of the link determines a map from the fundamental group of $M$ to $\mathbb{Z}$. This determines an infinite cyclic covering $\widetilde{M}$ of $M$ as well as a canonical generator $t$ for the corresponding $\mathbb{Z}$-action on the homology group $\mathcal{H}=H_{1}(\widetilde{M} ; \mathbb{Q})$. The Alexander polynomial is a palindromic polynomial $P(x) \in \mathbb{Z}[x]$ whose roots are the eigenvalues of the action of $t$ on $\mathcal{H}$.

Alexander polynomials form a rich testing ground for the minimization problem because of the following theorem.

Theorem 17 (Seifert [36]). $P(x) \in \mathbb{Z}[x]$ is a palindromic polynomial satisfying

$$
P(1)= \pm 1
$$

if and only if $P(x)$ is the Alexander polynomial of some knot $K$.

One is led to ask the following question.

Problem 18. Can the Mahler measure be minimized for monic non-cyclotomic Alexander polynomials?

We restrict our attention to a particular class of knots and links, called pretzel knots and links. To describe what they are, we start with the $(-2,3,7)$-pretzel knot. 


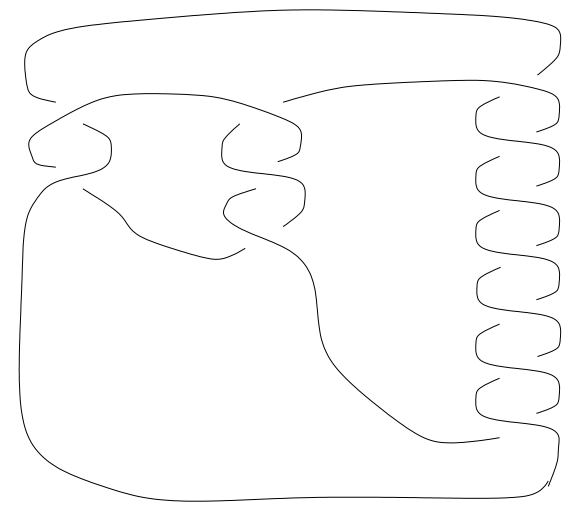

FiguRE 4.3. (-2,3,7)-pretzel knot

One takes 3 pairs of strings and twists them $-2,3$, and 7 times respectively. The negative sign with the 2 means a negative twist. Then one joins the top and bottom strands as in Figure 4.3.

By replacing $(-2,3,7)$ by any list of nonzero integers $\left(p_{1}, \ldots, p_{d}\right)$, we get a corresponding knot or link $L_{p_{1}, \ldots, p_{d}}$, called the $\left(p_{1}, \ldots, p_{d}\right)$-pretzel knot or link. If $p_{1}, \ldots, p_{d}$ are all odd, or at most one is even, then $L_{p_{1}, \ldots, p_{d}}$ is a knot; otherwise it is a link with number of components equal to one less than the number of even integers among $p_{1}, \ldots, p_{d}$. As described above any choice of orientations on the components of the link determines a one-variable Alexander polynomial for the link. This polynomial may be different for different choices of orientation.

Let $L(x)$ be Lehmer's polynomial. As Kirby points out in his problem list ([24], page 340$), L(-x)$ is the Alexander polynomial of the $(-2,3,7)$-pretzel knot, drawn in Figure 4.3. This seeming coincidence was also pointed out to the second author by D. Lind.

As mentioned in Section 4.1 Lehmer polynomial $L(x)$ is also the Salem polynomial $\Delta_{2,3,7}(x)$ which is the denominator of the growth series of the $(2,3,7)$ hyperbolic triangle group $G_{2,3,7}$. This leads naturally to the question: is $\Delta_{p_{1}, \ldots, p_{d}}(-x)$ also the Alexander polynomial of a pretzel knot or link? This question is answered by the following result.

Theorem 19 (Hironaka [22, Theorem 1.2). Let d be odd, and let $p_{1}, \ldots, p_{d}$ be positive integers. Then the Alexander polynomial of the $\left(p_{1}, \ldots, p_{d},-1\right)$-pretzel link, with respect to a suitable orientation of its components, is $\Delta_{p_{1}, \ldots, p_{d}}(-x)$.

This theorem applies to the $(-2,3,7)$-pretzel knot, which is equivalent to the $(2,3,7,-1)$-pretzel knot. The polynomials $\Delta_{p_{1}, \ldots, p_{d}}(-x)$ are related to the polynomials arising in Theorem 15. Since $M(P(x))=M(P(-x))$, for a monic polynomial $P(x) \in \mathbb{Z}[x]$, we have the following corollary.

Corollary 20. The minimum of the set of Mahler measures of Alexander polynomials of (suitably oriented) $\left(p_{1}, \ldots, p_{d},-1\right)$-pretzel links, as d varies through all odd integers and $p_{1}, \ldots, p_{d}$ through all positive integers, is attained by $\epsilon_{L}$, the Mahler measure of the $(2,3,7,-1)$-pretzel knot.

So far, questions about Salem numbers and Mahler measures arising from Alexander polynomials of more general links have not been fully addressed. Finding a 
concrete relation between the geometry of oriented knot and link complements and the Mahler measures of Alexander polynomials could lead to new insights into Problem 18 and to Lehmer's question itself.

\section{Special values of $L$-FunCtions}

We now continue our survey of the ubiquity of Salem numbers in mathematics by describing how they show up in expressions for the special values of $L$-functions.

5.1. Stark units and Salem numbers. Given an arbitrary Galois extension $K / k$ of number fields with group $G$ and a finite-dimensional complex representation $\rho: G \rightarrow G L(V)$, one has the Artin $L$-function

$$
L(s, K / k, \rho)=\prod_{p} \operatorname{det}\left(1-\left.F r_{p}\right|_{V^{I p}} N p^{-s}\right)^{-1},
$$

where the product runs over all (archimedean) primes $p$ of $k, I_{p}$ is an inertia subgroup at $p, V^{I_{p}}$ is the subspace of $V$ of $I_{p}$-fixed points, and $F r_{p}$ is a Frobenius element at $p$.

Stark has a conjectural description of the leading term in the Taylor expansion of this $L$-function at $s=0$, which states that it is essentially a certain $r \times r$ determinant, where $r$ is the order of vanishing of $L(s, K / k, \rho)$ at $s=0$. A precise statement can be found in [47]. When $r=1$, Stark's conjecture is known to be true (see 42]), and the above mentioned determinant is essentially just the logarithm of a special unit of $K$, now called a Stark unit.

We are interested in the case when $K / k$ is a Salem extension, hence of degree 2, and $\rho$ is the non-trivial quadratic character of $K / k$. In this case we have

$$
L(s, K / k, \rho)=\frac{\zeta_{K}(s)}{\zeta_{k}(s)},
$$

where $\zeta_{F}(s)$ is the Dedekind zeta function of the number field $F$. A formula of Dedekind says that the leading term in the Taylor expansion of $\zeta_{F}(s)$ at $s=0$ is given by

$$
\zeta_{F}(s)=\frac{h_{F} R_{F}}{w_{F}} s^{s_{F}+t_{F}-1}+\text { higher order terms, }
$$

where

- $h_{F}$ is the class number of $F$,

- $R_{F}$ is the regulator of $F$,

- $w_{F}$ is the number of roots of 1 in $F$, and,

- $s_{F}$ (respectively $t_{F}$ ) is the number of real (respectively complex) places of $F$.

Using this, we see that when $K / k$ is a Salem extension, $r=1$, and

$$
L^{\prime}(0, K / k, \rho)=\frac{h_{K} R_{K}}{h_{k} R_{k}} .
$$

The formula (4) is already very close to what is implied by Stark's conjecture. In fact, Stark showed:

Proposition 21 (Stark 42]). Let $K / k$ be a Salem extension. Then

$$
L^{\prime}(0, K / k, \rho)=\frac{h_{K} 2^{n-2} \log (e)}{h_{k} u}
$$


where $u=2$ when $K$ is generated over $k$ by a square root of a unit of $k$, and $u=1$ otherwise, and $e$ is a unit of $K$, which together with the units of $k$ generates a subgroup of index $2 u$ in $\mathcal{O}_{K}^{\times}$.

The relation between Stark's conjecture and our subject is seen by the following result.

Proposition 22 (Chinburg [12]). The unit e of $K$ is a Salem number. Conversely every Salem number $\epsilon$ in $K$ is of the form $\epsilon=e^{m / 2}$ for some positive integer $m$.

Chinburg also uses Proposition 22 to deduce some information on the relative class number $h_{K} / h_{k}$ of a Salem extension in [11].

5.2. Lower bounds for relative regulators. The formulas (4) and (5) of the previous section show that one might make some progress towards the minimization problem if one could establish a good absolute lower bound for the quotient of regulators $R_{K} / R_{k}$ corresponding to a Salem extension $K / k$. This observation was made by Costa, Friedman and Skoruppa, who in fact made the following general conjecture which for $r_{L / F}=1$ implies an affirmative answer to the minimization problem (see 21]):

Conjecture 23. Let $L / F$ denote an arbitrary extension of number fields, and let

$$
\operatorname{Reg}(L / F):=\frac{1}{\left[\mathcal{O}_{F}^{\times}: \mu_{F} N_{L / F}\left(\mathcal{O}_{L}^{\times}\right)\right]} \frac{R_{L}}{R_{F}},
$$

where $\mu_{F}$ is the group of roots of 1 in $F$. Then there are absolute constants $f_{0}$ and $f_{1}$ such that:

$$
\operatorname{Reg}(L / F) \geq f_{0} f_{1}^{r_{L / F}},
$$

where $r_{L / F} \geq 0$ denotes the difference in the unit ranks of $L$ and $F$.

Costa and Freidman prove Conjecture 23 when $[L: F]$ is 'large', and in the case when $L($ and so $F)$ is totally real (see [13]).

5.3. Mahler measures and $L$-values. The results of Section 5.1 point towards a connection between the (logarithms of) Mahler measures of polynomials and the special values of $L$-functions. In fact the first examples of this phenomena were discovered by Smyth for polynomials in more than one variables. Let $P\left(x_{1}, x_{2}, \ldots, x_{n}\right)$ $\in \mathbb{Z}\left[x_{1}, x_{2}, \ldots, x_{n}\right]$ be such a polynomial. Define its Mahler measure $M(P)$ by

$$
\log M(P)=\int \cdots \int_{S^{1} \times \cdots \times S^{1}}\left|\log P\left(x_{1}, \ldots, x_{n}\right)\right| \frac{d x_{1}}{x_{1}} \ldots \frac{d x_{n}}{x_{n}} .
$$

Using Jensen's formula one sees that when $n=1$ we recover the definition made in the Introduction. In [41] Smyth showed that

$$
\begin{gathered}
\log M(1+x+y)=L^{\prime}\left(\chi_{-3},-1\right), \\
\log M(1+x+y+z)=14 \zeta^{\prime}(-2),
\end{gathered}
$$

where $\chi_{-3}$ is the Dirichlet character attached to the quadratic extension $\mathbb{Q}(\sqrt{-3})$, and $\zeta(s)$ is just the Riemann zeta function. Other striking examples of this kind were given by Ray 32 .

The first 'non-abelian' example is due to Deninger [15] and Boyd [9]. They showed

$$
\log M\left(y^{2}+\left(x^{2}+x+1\right) y+x^{2}\right)=L^{\prime}\left(E_{15}, 0\right)
$$


where $E_{15}$ is the elliptic curve of conductor 15 defined by the polynomial appearing in (6) above. Actually this formula has only been checked to 50 decimal places of accuracy, though in [15] Deninger has been able to give a heuristic reason for its validity. Moreover in some cases Deninger (see also [3]) has been able to interpret Mahler measures as Deligne periods of mixed motives.

Many other formulae similar to (6) have been numerically identified by Boyd [9], and via different methods, by Rodriguez Villegas [50]. Interestingly, proofs, and not just numerical coincidence, of these formulae have so far been forthcoming mostly for curves $E$ with complex multiplication, in which case both the $L$-value and the Mahler measure reduce to the value of an Eisenstein-Kronecker series evaluated at the corresponding point of complex multiplication. We refer the reader to the papers mentioned above for more details.

In closing this section we would like to point out a curious discovery of Boyd [9]. Boyd noticed that there is a formula similar to (6) above for $L^{\prime}\left(E_{14}, 0\right)$ where $E_{14}$ is the elliptic curve over $\mathbb{Q}$ with conductor 14 :

$$
\begin{aligned}
\log M\left((x+1) y^{2}+\left(x^{2}+x+1\right) y+\left(x^{2}+x\right)\right) & =0.2274812230 \ldots \\
& =L^{\prime}\left(E_{14}, 0\right) .
\end{aligned}
$$

This formula has again only been checked numerically. Since we have (see [8])

$$
\lim _{m \rightarrow \infty} M\left(P\left(x, x^{m}\right)\right)=M(P(x, y)),
$$

we see that $\exp \left(L^{\prime}\left(E_{14}, 0\right)\right)=1.25543 \ldots$ is a (potential) limit point of the set of Mahler measures of polynomials in one variable. As it turns out, it is the smallest known limit point.

Now apart from $E_{14}$ there is, up to isogeny, exactly one more elliptic curve over $\mathbb{Q}$ with conductor smaller than 15 , namely the curve $E_{11}$ of conductor 11 . Yet no such analogous formula has so far been discovered for $E_{11}$. Since $\exp \left(L^{\prime}\left(E_{11}, 0\right)\right)=$ $1.16433 \ldots<\epsilon_{L}=1.17628 \ldots$, the existence of such a formula for $E_{11}$ would imply that one would be able to find infinitely many polynomials in one variable, with Mahler measure smaller than that of Lehmer's polynomial $L(x)$.

\section{Best RESUlts AND RECORDS}

This section contains the best results (known to us) concerning lower bounds on Salem numbers and Mahler measures of irreducible monic polynomials $P(x) \in \mathbb{Z}[x]$ in one variable.

In 1979, Dobrowolski [16] obtained a lower bound $B(d)$ for the Mahler measure of polynomials of degree $d$. Since then, his methods have been refined by various mathematicians. As Boyd points out [8] the existence of $B(d)$ is not in question since as we have seen (see the proof of Lemma 3) the set of Mahler measures of monic polynomials with integer coefficients of a fixed degree $d$ has a minimal element. In any case, a sample of such a result is the

Theorem 24 (Voutier, [51]). If $P(X)$ is not a cyclotomic polynomial and has degree $d>1$, then

$$
\log M(P)>\frac{1}{4}\left(\frac{\log (\log (d))}{\log (d)}\right)^{3} .
$$

Note that the bound above tends to 0 as $d \rightarrow \infty$. 
One also has bounds that depend on the number of real roots of $P(X)$. For instance it seems that a lower bound for $M(P)$ for those polynomials $P(X)$ which have at least one non-real root outside the unit circle is $1.2013 \ldots$ This is the Mahler measure of the polynomial (cf. [7]):

$$
x^{18}+x^{17}+x^{16}-x^{13}-x^{11}-x^{9}-x^{7}-x^{5}+x^{2}+x^{1}+1
$$

which has two complex-conjugate roots outside the unit circle. In this vein we have the following theorem (cf. 27, page 322).

Theorem 25 (Laurent). Let $P(X)$ be a non-cyclotomic polynomial, with $r$ real roots, of degree $d$. Then

$$
\log M(P) \geq c \frac{r^{2}}{d \log (1+d / r)},
$$

where $c>0$ is an absolute constant.

Note that for $r=2$, which is the minimization problem for Salem numbers, this is a better bound than the one above, yet it still tends to 0 as $d \rightarrow \infty$.

In closing, we refer the reader to the very informative web page on Lehmer's Conjecture maintained by Mossinghoff [28], where one may find many lists and records related to Problems 1 and 2 For instance, there are tables of the smallest hundred Mahler measures known, the smallest known Mahler measure of each degree $d \leq 180$, the smallest known Mahler measure of a given height $(=$ the maximum of the absolute values of the coefficients), the known forty-seven Salem numbers less than 1.3 and so on and so forth. There is also a bibliography on Lehmer's conjecture there which nicely complements our own.

\section{REFERENCES}

[1] E. Artin and J. Tate, Class field theory, Harvard notes, W.A. Benjamin, Inc., New York, 1967. MR 36:6383

[2] M.-J. Bertin, A. Decomps-Guilloux, M. Grandet-Hugot, M. Pathiaux-Delefosse, and J.P. Schreiber, Pisot and Salem numbers, Birkhauser Verlag, Basel, 1992. MR 93k:11095

[3] H. Bornhorn, Mahler-masse und speziell werte von L-funktionen, Preprintreihe, SFB 478 Geometrische Strukturen in der Mathematik ISSN 1435-1188 (1999).

[4] N. Bourbaki, Groupes et algèbres de Lie, Fasc. XXXIV, vol. 1337, Hermann, Paris, 1968. MR 39:1590

[5] D. W. Boyd, Small salem numbers, Duke Math. J. 44 (1977), no. 2, 315-328. MR 56:11952

[6] _ Pisot and salem numbers in intervals of the real line, Math. Comp. 32 (1978), no. 144, 1244-1260. MR 58:10812

[7] _ Reciprocal polynomials having small measure, Math. Comp 35 (1980), no. 152, 13611377. MR 82a:30005

[8] _ Speculations concerning the range of Mahler's measure, Canad. Math. Bull. 24 (1981), no. 4, 453-469. MR 83h:12002

[9] _ Mahler's measure and special values of L-functions, Experimental Math. 7 (1998), no. 1, 37-82. MR 99d:11070

[10] J. W. Cannon and Ph. Wagreich, Growth functions of surface groups, Math. Ann. 292 (1992), 239-257. MR 93j:20077

[11] T. Chinburg, On the arithmetic of two constructions of Salem numbers, J. Reine Angew. Math. 348 (1984), 166-179. MR 85h:11056

[12] _ Salem numbers and L-functions, J. Number Theory 18 (1984), no. 2, 213-214. MR 85i: 11089

[13] A. Costa and E. Friedman, Ratios of regulators in totally real extensions of number fields, J. Number Theory 37 (1991), 288-297. MR 92j:11138

[14] H.S.M. Coxeter and W.O.J. Moser, Generators and relations for discrete groups, SpringerVerlag, Berlin, 1980. MR 81a:20001 
[15] C. Deninger, K-theory, mixed motives, and the entropy of $\mathbb{Z}^{n}$-actions, J. of the A.M.S. 10 (1997), no. 2, 259-281. MR 97k:11101

[16] E. Dobrowolski, On a question of Lehmer and the number of irreducible factors of a polynomial, Acta. Arith. 34 (1979), no. 4, 391-401. MR 80i:10040

[17] G. Everest and T. Ward, Heights of polynomials and entropy in algebraic dynamics, Universitext, Springer-Verlag London, Ltd., London, 1999. MR 2000e:11087

[18] V. Flammang, M. Grandcolas, and G. Rhin, Small Salem numbers, Number theory in progress, Zakopane-Kościelisko, 1997, de Gruyter, Berlin, 1999, pp. 165-168. MR 2000e:11132

[19] W. J. Floyd and S. P. Plotnick, Symmetries of planar growth functions of Coxeter groups, Invent. Math. 93 (1988), 501-543. MR 89f:22016

[20] R. H. Fox, A quick trip through knot theory, Topology of 3-manifolds, Prentice-Hall, 1962, pp. 120-167. MR 25:3522

[21] E. Friedman and N-P. Skoruppa, Relative regulators of number fields, Invent. Math. 135 (1999), 115-144. MR 2000c:11187

[22] E. Hironaka, The Lehmer polynomial and pretzel knots, Bull. of Canadian Math. Soc. (to appear) (1998).

[23] N. Jacobson, Basic Algebra II, Second Edition, W. H. Freeman and Company, New York, 1989. MR 90m:00007

[24] R. Kirby, Problems in low-dimensional topology, Geometric Topology (W. H. Kazez, ed.), Studies in Advanced Mathematics, A.M.S., 1997. CMP 98:01

[25] D. H. Lehmer, Factorization of certain cyclotomic functions, Ann. of Math. 34 (1933), 461469.

[26] D. Lind, K. Schmidt, and T. Ward, Mahler measure and entropy for commuting automorphisms of compact groups, Invent. Math. 101 (1990), no. 3, 593-629. MR 92j:22013

[27] G. A. Margulis, Discrete subgroups of semisimple Lie groups, Springer Verlag, 1991. MR 92h:22021

[28] M. J. Mossinghoff, Lehmer's Conjecture, http://www.math.ucla.edu/ ${ }^{\sim}$ mjm.

[29] _ Polynomials with small Mahler measure, Math. Comp. 67 (1998), no. 3, 1697-1705. MR 99a:11119

[30] W. D. Neumann and A. W. Reid, Arithmetic of hyperbolic manifolds, Topology '90 (B. Apanasov, W. D. Neumann, A. W. Reid, and L. Siebenmann, eds.), Walter de Gruyter and Co., Berlin, 1992, pp. 273-310. MR 94c:57024

[31] W. Parry, Growth series of Coxeter groups and Salem numbers, J. of Alg. 154 (1993), 406415. MR 94e:20043

[32] G. A. Ray, Relations between Mahler's measure and values of L-series, Can. J. Math. 39 (1987), 694-732. MR 88m:11071

[33] D. Rolfsen, Knots and links, Publish or Perish, Inc, Berkeley, 1976. MR 58:24236

[34] R. Salem, Algebraic numbers and Fourier analysis, Heath, Boston, MA, 1963. MR 28:1169]

[35] K. Schmidt, Dynamical systems of algebraic origin, Progress in Mathematics, 128, Birkhauser Verlag, Basel, 1995. MR 97c:28041

[36] H. Seifert, Über das geschlecht von knoten, Math. Ann. 110 (1934), 571-592.

[37] J.-P. Serre, Cohomologie des groupes discrets, Prospects in Mathematics, Ann. Math. Stud., vol. 70, Princeton University Press, 1971, pp. 77-169. MR 52:5876

[38] _ Local fields, GTM 67, Springer-Verlag, Berlin-New York, 1979. MR 82e:12016

[39] J. Silverman, Small Salem numbers, exceptional units, and Lehmer's conjecture, Symposium on Diophantine Problems (Boulder, CO, 1994), Rocky Mountain J. Math. 26 (1996), no. 3, 1099-1114. MR 97k:11152

[40] C. J. Smyth, On the product of the conjugates outside the unit circle of an algebraic integer, Bull. London Math. Soc. 3 (1971), 169-175. MR 44:6641

[41] - On measures of polynomials in several variables, Bull. Austral. Math. Soc. 23 (1981), 49-63. MR 84g:10088

[42] H. Stark, L-functions at $s=1$, II, Advan. in Math. 17 (1975), 60-92. MR 52:3082

[43] R. Steinberg, Finite reflection groups, Trans. Amer. Math. Soc. 91 (1959), 493-504. MR 21:5160

[44] B. Sury, Letter to D. Boyd, (1983).

[45] — Arithmetic groups and Salem numbers, Manuscr. Math. 75 (1992), no. 1, 97-102. MR 92m:11042 
[46] K. Takeuchi, Commensurability classes of arithmetic triangle groups, Fac. Sci. Univ. Tokyo 24 (1977), no. 1, 201-212. MR 57:3077

[47] J. Tate, Les conjectures de Stark sur les fonctions L d'Artin en $s=0$, Birkhäuser, 1984. MR 86e: 11112

[48] W. Thurston, The geometry and topology of 3-manifolds, (unpublished lecture notes), 1977.

[49] M.-F. Vignéras, Arithmetique des algèbres de quaternions, Springer-Verlag LNM 800 (1984). MR 82i:12016

[50] F. Rodriguez Villegas, Modular Mahler measures I, Topics in Number Theory, 1997 (S.D. Ahlgren, G. E. Andrews, and K. Ono, eds.), Mathematics and its Applications, vol. 467, Kluwer Academic Publishers, Dordrecht, 1999. MR 2000e:11085

[51] P. Voutier, An effective lower bound for the height of algebraic numbers, Acta. Arith. 74 (1996), no. 1, 81-95. MR 96j:11098

School of Mathematics, Tata Institute of Fundamental Research, Homi Bhabha Road, Mumbai, 400 005, India

E-mail address: eghate@math.tifr.res.in

Department of Mathematics, Florida State University, Tallahassee, FL 32306

E-mail address: hironaka@math.fsu.edu 